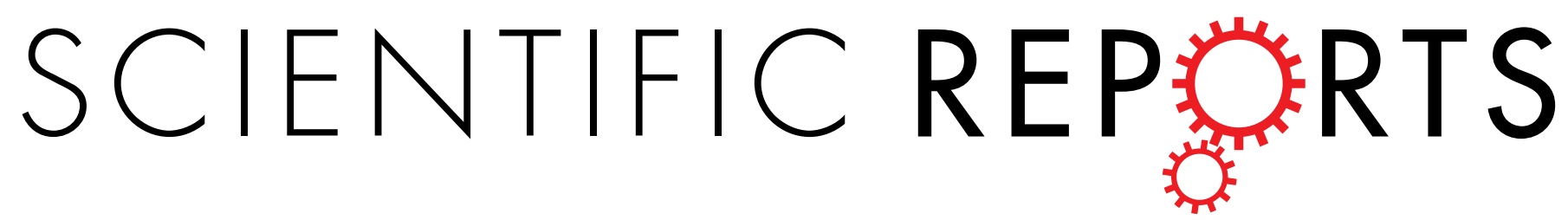

\title{
Erratum: Moisture-responsive films of cellulose stearoyl esters showing reversible shape transitions
}

Kai Zhang, Andreas Geissler, Michaela Standhardt, Sabrina Mehlhase, Markus Gallei, Longquan Chen \& Christina Marie Thiele

Scientific Reports 5:11011; doi: 10.1038/srep11011; published online 08 June 2015; updated on 05 August 2015

This Article contains a typographical error in the affiliation of Longquan Chen. The correct affiliation is listed below:

School of Mechanics and Engineering, Southwest Jiaotong University, 610031, Chengdu China. 